\title{
Pregnancy-induced gene expression changes in vivo among women with rheumatoid arthritis: a pilot study
}

Dana E. Goin ${ }^{1,2}$, Mette Kiel Smed ${ }^{3}$, Lior Pachter ${ }^{2,4}$, Elizabeth Purdom², J. Lee Nelson ${ }^{5,6}$, Hanne Kjærgaard ${ }^{\wedge}$, Jørn Olsen ${ }^{7,8}$, Merete Lund Hetland ${ }^{9,10}$, Vibeke Zoffmann ${ }^{3,10}$, Bent Ottesen ${ }^{3}$ and Damini Jawaheer ${ }^{1,8,11^{*}}$

\begin{abstract}
Background: Little is known about gene expression changes induced by pregnancy in women with rheumatoid arthritis (RA) and healthy women because the few studies previously conducted did not have pre-pregnancy samples available as baseline. We have established a cohort of women with RA and healthy women followed prospectively from a pre-pregnancy baseline. In this study, we tested the hypothesis that pregnancy-induced changes in gene expression among women with RA who improve during pregnancy (pregDASimproved) overlap substantially with changes observed among healthy women and differ from changes observed among women with RA who worsen during pregnancy (pregDAS ${ }_{\text {worse }}$ ).
\end{abstract}

Methods: Global gene expression profiles were generated by RNA sequencing (RNA-seq) from 11 women with RA and 5 healthy women before pregnancy (T0) and at the third trimester (T3). Among the women with RA, eight showed an improvement in disease activity by T3, whereas three worsened. Differential expression analysis was used to identify genes demonstrating significant changes in expression within each of the RA and healthy groups (T3 vs T0), as well as between the groups at each time point. Gene set enrichment was assessed in terms of Gene Ontology processes and protein networks.

Results: A total of 1296 genes were differentially expressed between T3 and T0 among the 8 pregDAS improved women, with 161 genes showing at least two-fold change (FC) in expression by T3. The majority (108 of 161 genes) were also differentially expressed among healthy women $(q<0.05, F C \geq 2)$. Additionally, a small cluster of genes demonstrated contrasting changes in expression between the pregDAS improved and pregDAS worse groups, all of which were inducible by type I interferon (IFN). These IFN-inducible genes were over-expressed at T3 compared to the TO baseline among the pregDASimproved women.

Conclusions: In our pilot RNA-seq dataset, increased pregnancy-induced expression of type I IFN-inducible genes was observed among women with RA who improved during pregnancy, but not among women who worsened. These findings warrant further investigation into expression of these genes in RA pregnancy and their potential role in modulation of disease activity. These results are nevertheless preliminary and should be interpreted with caution until replicated in a larger sample.

Keywords: Rheumatoid arthritis, Pregnancy, RNA-seq, Gene expression, Type I interferon

\footnotetext{
*Correspondence: djawaheer@choriorg

Deceased

'UCSF Benioff Children's Hospital Oakland, Children's Hospital Oakland

Research Institute, 5700 Martin Luther King Jr. Way, Oakland, CA, USA

${ }^{8}$ Aarhus University, Aarhus, Denmark

Full list of author information is available at the end of the article
} 


\section{Background}

Rheumatoid arthritis (RA) is a systemic inflammatory disease characterized by inflammation of the joints that continues to contribute significantly to the global burden of disease [1]. To date, its etiopathogenesis remains unknown, and despite major advancements in treatment, a cure remains elusive. Intriguingly, however, it is well documented that pregnancy can induce significant changes in RA disease activity. During pregnancy, 50$75 \%$ of women with RA experience a natural and often dramatic improvement in disease, whereas others may worsen or show no change [2-5]. Some pregnancyrelated factors, including maternal-fetal disparity at the human leukocyte antigen locus [6] and fetal microchimerism $[7,8]$, have been associated with the pregnancyinduced improvement of RA symptoms. Nonetheless, the biology of human pregnancy in terms of the overall systemic changes that it induces, how those may differ between RA and healthy women, and how the pregnancy-induced changes may have an impact on RA remain unknown.

Gene expression studies, more specifically comparison of gene expression profiles (third trimester vs prepregnancy baseline) from women followed longitudinally from the pre-pregnancy state to the third trimester, can provide information about biological changes that are induced during pregnancy. Unfortunately, there have been no gene expression studies in which women - healthy or with RA - were studied before they became pregnant as well as during pregnancy to examine such pregnancy-induced changes. Only few studies of gene expression have been conducted in the context of RA pregnancy, using microarray technology [9-12]. In these studies, pregnancy-related changes with respect to the pre-pregnancy state were not examined, due to pre-pregnancy samples not being available [9-11], or pregnancy profiles were compared to profiles of unrelated non-pregnant women [12].

We have established a cohort of RA and healthy women enrolled before pregnancy and followed prospectively through pregnancy. Using state-of-the-art RNA sequencing (RNA-seq) technology, we examined systemic global gene expression in peripheral blood in a subset of women from this cohort as a pilot. We previously identified genes whose expression are modulated by pregnancy in both RA and healthy women, and we showed that a few genes are modulated differently in RA compared to healthy women [13]. We have now used differential expression analysis to further investigate pregnancy-induced gene expression patterns and foldchanges (FCs) in expression within our pilot dataset, taking into account whether the women with RA improved or worsened during pregnancy. We hypothesized that pregnancy-induced changes in gene expression among women with RA who improve during pregnancy may overlap substantially with those observed among healthy women and may differ from changes among women with RA who worsen during pregnancy.

\section{Methods \\ Study subjects}

Women with RA and healthy women of Danish descent were recruited and enrolled in a pregnancy cohort in Denmark, as previously described [13]. A subset of 20 women with RA and 5 healthy women from this cohort were included in the pilot dataset. Subjects with RA fulfilled the 1987 revised American College of Rheumatology criteria for RA [14]. The study was approved by the ethics committee for Region Hovedstaden (Denmark), the Danish Data Protection Agency, and the Children's Hospital Oakland Research Institute Institutional Review Board. All subjects provided written informed consent prior to enrollment.

\section{Data}

Data collected before pregnancy (T0) and at the third trimester (T3) were included in the present study. Therefore, women who had missing data at the T0 baseline or at T3 (eight women with RA) were excluded from further analyses. At both time points, the women with RA were examined by trained study nurses. Disease activity measures including tender and swollen joint counts based on a total of 28 joints (TJC28 and SJC28, respectively) and patient global health $(\mathrm{GH})$ scores were recorded, and a serum sample was collected for measurement of C-reactive protein (CRP) levels. Data on medication use during the 3 months prior to both visits were also recorded. At each visit, blood samples from the women with RA and healthy women were collected in PAXgene RNA tubes (PreAnalytiX, Hombrechtikon, Switzerland).

\section{Assessment of RA disease activity}

RA disease activity was assessed using the Disease Activity Score based on 28 joints and 4 variables (DAS28CRP4, abbreviated henceforth as DAS28), as follows [15]:

$$
\begin{aligned}
\text { DAS28 }= & (0.56 * \sqrt{T J C 28})+(0.28 * \sqrt{S J C 28}) \\
& +(0.36 * \ln (C R P+1))+0.014 * \mathrm{GH}+0.96
\end{aligned}
$$

Change in DAS28 from T0 to T3 was calculated as: $\triangle \mathrm{DAS} 28=$ DAS$^{2} 8_{\mathrm{T} 3}-\mathrm{DAS} 8_{\mathrm{T} 0}$.

The women with RA were categorized into two subsets: those with negative $\triangle \mathrm{DAS} 28$ values were considered to show an improvement in DAS28 score at T3 compared to the T0 baseline (referred to as the 


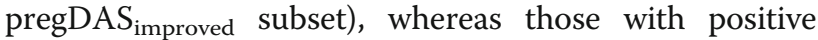
$\triangle$ DAS28 values were included in the "worsened" subset,

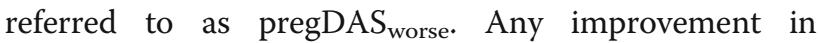
DAS28 scores $(\triangle \mathrm{DAS} 28<0)$ was used as a surrogate for improvement in disease activity. This was done to capture even small changes in disease activity that may correlate with biological changes observed at the gene expression level. Disease activity categories (remission, low, moderate, or high) were assessed using previously defined criteria [16].

\section{Sample processing and bioinformatic analyses}

Total RNA was isolated from frozen blood samples, and barcoded cDNA libraries were prepared as previously described [13]. Pseudo-alignment of the de-multiplexed raw sequence reads (FASTQ format) to the Ensembl reference human GRCh38 transcriptome assembly, and quantification of transcript abundances was performed using kallisto (version 0.42.4) [17]. BioMart annotations were used to combine transcript-level counts into genelevel estimates. Pseudogenes, genes with no annotations, and genes with very low read counts $(<1$ count per million) in at least $25 \%$ of all samples were filtered out. Any globin genes and ribosomal RNA (rRNA) transcripts still present were also filtered out. To adjust for variable sequencing depths across samples, the gene-level counts were normalized using the Trimmed Mean of M values (TMM) algorithm as implemented in the edgeR package (version 3.10.5) [18, 19]. To assess batch effects, normalized gene counts from each pair of technical replicates were plotted, and correlations were assessed.

\section{Statistical analyses}

Differential gene expression analysis was performed using edgeR (version 3.10.5) [18] to compare normalized gene-level counts between the T3 and T0 time points within each group of women (i.e., pregDAS $S_{\text {im- }}$ proved, pregDAS ${ }_{\text {worse }}$ and healthy women). A model with a paired-sample design was used for these within-group comparisons. Between-group comparisons (pregDAS $\mathrm{im}_{\mathrm{im}}$ proved vs pregDAS ${ }_{\text {worse }}$, and pregDAS $S_{\text {improved }}$ vs healthy) at each time point were also performed using differential expression analyses. A negative binomial distribution was used to handle the over-dispersion in RNAseq gene counts. Differential expression was tested using generalized linear model (GLM) likelihood ratio tests. To correct for any batch effects, batch was included as a covariate in the model. A $q$ value threshold of 0.05 was used to assess significance. Because sample sizes were small, FCs in expression were also used in the interpretation of results, focusing on genes with at least a two-fold change in expression from T0 to T3.

\section{Functional analysis}

Differentially expressed genes were analyzed for overrepresentation of Gene Ontology (GO) categories using a hypergeometric test implemented in the Web-based Gene Set Analysis Toolkit (WebGestalt) with a threshold of at least five genes per category [20]. A significance threshold of $\mathrm{q}<0.05$ was used to define enrichment. Functional enrichment of gene sets was examined using the STRING database of known and predicted interactions among proteins $[21,22]$.

\section{Results}

\section{Study subjects}

Of the 12 women with RA who had data at both T0 and T3, 8 were in the pregDAS $S_{\text {improved }}$ group and 3 were in the pregDAS ${ }_{\text {worse }}$ group. One woman was excluded because, although she had an increase in DAS28 at T3, she was in remission at both time points and hence did not fit into the pregDAS ${ }_{\text {worse }}$ group. The DAS28 scores of the 11 women included in the analyses are shown in Fig. 1 . The average disease duration among the women with RA was (mean $\pm \mathrm{SD}$ ) $5.9 \pm 4.4$ years for pregDAS improved $_{\text {and }} 8.7$ \pm 1.0 years for pregDAS ${ }_{\text {worse }}$. The average age at conception was $30.3 \pm 5.7$ years for pregDAS $S_{\text {improved }}, 33.2 \pm 1.9$ years for pregDAS ${ }_{\text {worse }}$, and $31.2 \pm 5.7$ years for the healthy women.

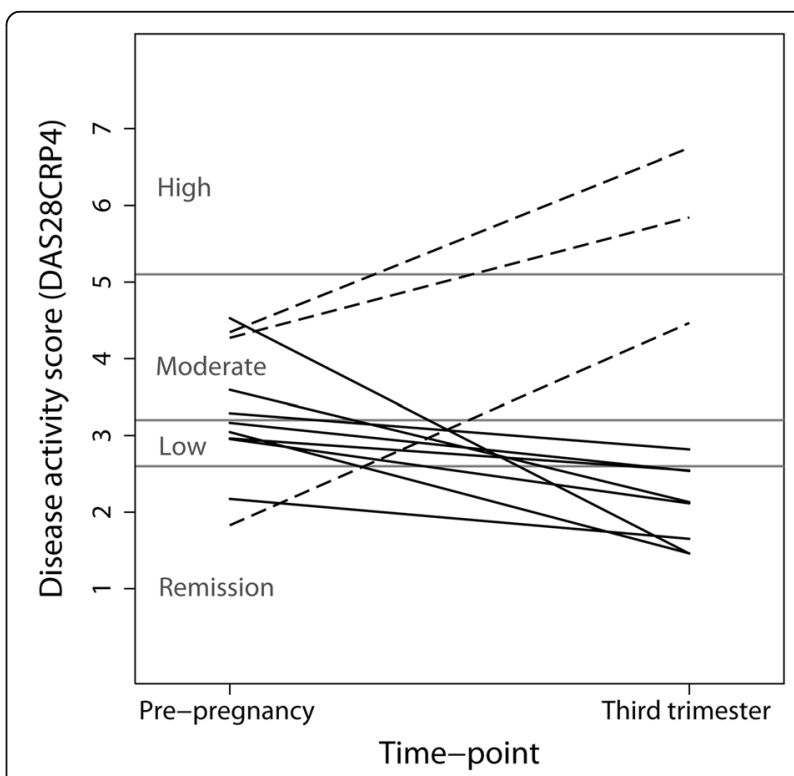

Fig. 1 Disease activity before pregnancy and at the third trimester among the women with rheumatoid arthritis. Disease Activity Scores based on 28 joints and 4 variables (DAS28-CRP4) are shown for the eight women who improved during pregnancy (solid lines) and the three women who worsened (dashed lines), at the pre-pregnancy baseline and at the third trimester. (The straight lines between time-points are included for graphical purposes only and do not imply a linear change in score.) 
Among the eight pregDAS improved $_{\text {women, three did }}$ not take any medications at T0 and only one of these three women started taking medications by T3 (prednisolone + sulfasalazine). The remaining five women were taking prednisolone and/or sulfasalazine at both time points; one of them was also on anti-tumor necrosis factor (anti-TNF) therapy at T0, and another was taking methotrexate at T0. The three pregDAS ${ }_{\text {worse }}$ women were all on anti-TNF therapy and taking prednisolone and/or sulfasalazine (except for one) at T0; at T3, one was taking prednisolone + sulfasalazine, one remained on anti-TNF therapy, and one stopped taking medications.

\section{Genes differentially expressed between T3 and T0 in the pregDAS $_{\text {improved }}$ subset}

A total of 1296 genes showed significant differential expression between $\mathrm{T} 3$ and $\mathrm{T} 0$ in the pregDAS $\mathrm{S}_{\text {improved }}$ subset, of which 161 displayed two-fold or more change in expression (see Additional file 1). These 161 genes were enriched in a number of immune-related pathways, as shown in Table 1. Similar results were obtained when the woman who was receiving anti-TNF therapy at T0 was excluded from the analysis.

\section{Overlap with genes differentially expressed among healthy women}

A large proportion of the 161 genes that exhibited at least two-fold change in expression between T3 and T0

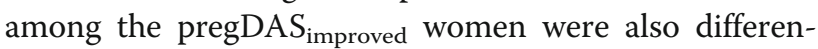
tially expressed among healthy women $(q<0.05)$, mostly $(n=108)$ with two-fold or more change in expression (see Additional files 1 and 2), although some $(n=33)$ showed

Table 1 Gene Ontology (GO) biological processes enriched in genes differentially expressed among women with RA who improved during pregnancy

\begin{tabular}{lll}
\hline GO biological process & Gene count & 9 value \\
\hline Immune system process & 55 & $7.9 \times 10^{-14}$ \\
Response to stress & 54 & $9.1 \times 10^{-6}$ \\
Defense response & 35 & $1.5 \times 10^{-8}$ \\
Immune response & 34 & $2.2 \times 10^{-8}$ \\
Multiorganism process & 34 & $6.7 \times 10^{-6}$ \\
Response to other organism & 27 & $1.4 \times 10^{-9}$ \\
Response to biotic stimulus & 27 & $2.9 \times 10^{-9}$ \\
Innate immune response & 22 & $5.6 \times 10^{-7}$ \\
Response to bacterium & 16 & $9.0 \times 10^{-6}$ \\
Erythrocyte differentiation & 10 & $6.5 \times 10^{-7}$ \\
\hline
\end{tabular}

Of the 161 genes differentially expressed (with $\mathrm{FC} \geq 2$ ) between $\mathrm{T} 0$ and $\mathrm{T} 3$ among pregDAS $S_{\text {improved }}$ women, several were enriched in $\mathrm{GO}$ biological processes relating to immune functions. (Note: These 161 genes included some that were also differentially expressed among healthy women and pregDAS ${ }_{\text {worse }}$ women.) more modest $\mathrm{FCs}$ in expression $(1.5 \leq \mathrm{FC}<2.0)$. In addition, another 77 genes differentially expressed $(q<0.05)$ in both the pregDAS improved $_{\text {and healthy groups }}$ showed lower FCs among the pregDAS $S_{\text {improved }}$ women $(1.5 \leq \mathrm{FC}<2.0)$ than among the healthy women $(\mathrm{FC} \geq 2)$. The genes with $\mathrm{FC} \geq 2$ in both the women with $\mathrm{RA}$ and healthy women were enriched in immune-related pathways such as immune system process $\left(q=7.9 \times 10^{-9}\right)$, response to other organism $\left(q=2.7 \times 10^{-7}\right)$, and defense response to bacterium $\left(q=9.3 \times 10^{-6}\right)$.

\section{Overlap with genes differentially expressed in the pregDAS ${ }_{\text {worse }}$ subset}

Of the 161 genes differentially expressed in the pregDA$S_{\text {improved }}$ subset, some $(n=31)$ were also differentially expressed $(q<0.05, \mathrm{FC} \geq 2)$ among pregDAS ${ }_{\text {worse }}$ women, with most of these genes overlapping with those differentially expressed among healthy women $(n=30)$ (see Additional file 2). The FCs in expression from T0 to T3 were correlated between the two RA subsets (Pearson correlation coefficient $r=0.86, p<0.00005$ ).

\section{Differences in genes differentially expressed (T3 vs T0)} between the pregDAS improved $_{\text {and pregDAS }}$ worse subsets The remaining 130 genes that were differentially expressed (T3 vs T0: $\mathrm{FC} \geq 2, q<0.05$ ) in our data among the pregDAS $S_{\text {improved }}$ women, but not among the pregDAS $_{\text {worse }}$ women, were significantly enriched in several immune-related $\mathrm{GO}$ processes, such as immune system process $\left(q=4.7 \times 10^{-10}\right)$, defense response $\left(q=9.6 \times 10^{-6}\right)$, immune response $\left(q=5.0 \times 10^{-5}\right)$, and innate immune response $\left(q=8.0 \times 10^{-6}\right)$, among others (Table 2). Of these 130 genes, 78 were also differentially expressed among

Table 2 Gene Ontology biological processes enriched in genes differentially expressed among women with RA who improved during pregnancy, but not among those who worsened

\begin{tabular}{lll}
\hline GO biological process & Gene count & q value \\
\hline Immune system process & 43 & $4.7 \times 10^{-10}$ \\
Defense response & 26 & $9.6 \times 10^{-6}$ \\
Immune response & 24 & $5.0 \times 10^{-5}$ \\
Innate immune response & 18 & $8.0 \times 10^{-6}$ \\
Response to other organism & 17 & $6.7 \times 10^{-5}$ \\
Response to biotic stimulus & 17 & $1.0 \times 10^{-4}$ \\
Hemopoiesis & 15 & $2.0 \times 10^{-4}$ \\
Homeostasis of number of cells & 11 & $6.9 \times 10^{-6}$ \\
Myeloid cell differentiation & 11 & $7.1 \times 10^{-5}$ \\
Erythrocyte differentiation & 10 & $2.5 \times 10^{-7}$ \\
\hline
\end{tabular}

The 130 genes that were differentially expressed (with $\mathrm{FC} \geq 2$ ) between $\mathrm{T} 0$ and T3 among pregDAS ${ }_{\text {improved, }}$ but not among pregDAS worse $_{\text {women, included }}$ several that were enriched in $\mathrm{GO}$ biological processes relating to immune functions 
healthy women (see Additional files 1 and 2). A comparison of the FCs in expression from T0 to T3 within each of the RA subsets revealed a small cluster of genes that were significantly over-expressed at T3 among pregDAS $S_{\text {improved }}$ women and under-expressed (FC $\geq 2$, not significant) among pregDAS ${ }_{\text {worse }}$ women (Fig. 2). By T3, mean normalized expression levels of these genes were higher among the pregDAS $S_{\text {improved }}$ women than among the pregDAS ${ }_{\text {worse }}$ women. Interestingly, all of the genes in this cluster were type I interferon (IFN)-inducible (IFI44, IFI44L, IFIT1, HERC5, $C M P K 2, R S A D 2$, and SIGLEC1). Among these, only the RSAD2 gene was differentially expressed (underexpressed) at $\mathrm{T} 3$ compared to $\mathrm{T} 0$ among healthy women $(q<0.05, \mathrm{FC} \geq 2)$. A number of other type I IFNinducible genes (STAT1, IFI16, IFIT2, IFIT5, IFIH1, and $M X 1)$ also exhibited similar patterns of expression, being over-expressed at T3 $(q<0.05)$ among pregDAS $S_{\text {improved }}$ women, though FCs were more modest (1.5- to 1.9-fold), and under-expressed among pregDAS ${ }_{\text {worse }}$ women. The PLSCR1 gene, which can enhance the type I IFN response,

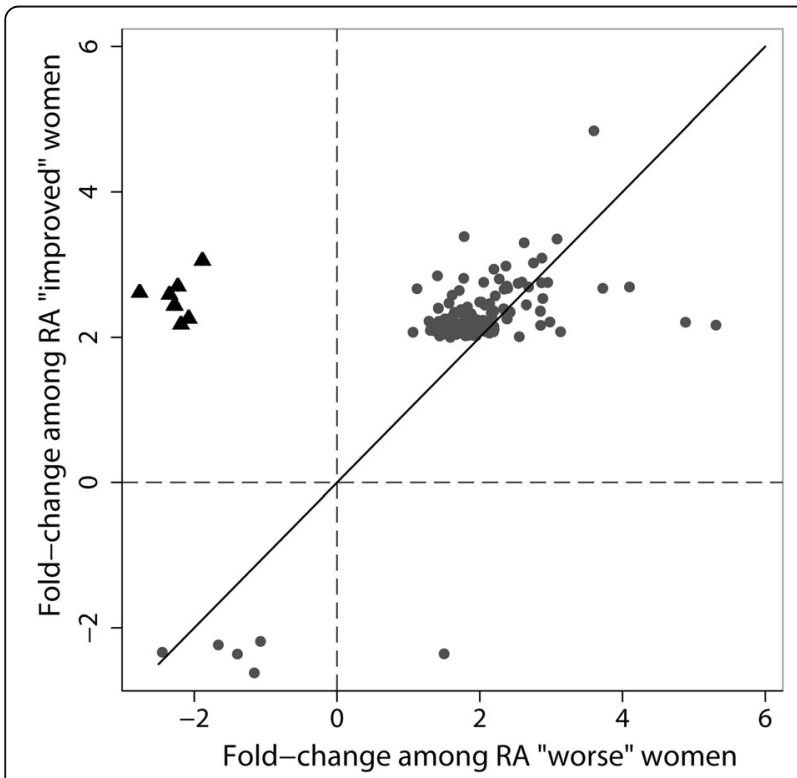

Fig. 2 Fold-changes in expression for genes differentially expressed (third trimester [T3] vs baseline [T0]) among women who improved, but not among those who worsened. Fold-changes in expression are plotted for women who improved during pregnancy ( $y$-axis) and women who worsened during pregnancy ( $x$-axis) only for those genes that were differentially expressed (T3 vs T0) in the "improved" group $(q<0.05)$ and not in the "worsened" group. Only genes with $F C \geq 2$ are shown. Fold-changes for genes that were under- expressed at $\mathrm{T} 3$ are shown as negative values. The majority of genes showed similar expression changes in both groups of women with RA, as shown by the gray dots around the $y=x$ line (solid line). A small cluster of genes (black triangles), however, was over-expressed among women who improved and under-expressed among women who worsened. Genes in this cluster were IFI44, IFI44L, IFIT1, HERC5, CMPK2, RSAD2, and SIGLEC1 also showed a significant increase in expression $(\mathrm{FC} \geq 2)$ in

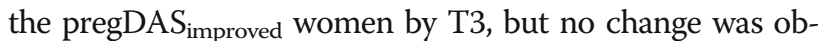
served in the pregDAS ${ }_{\text {worse }}$ women. Of interest, a comparison of normalized expression levels of these genes at T0 between pregDAS $S_{\text {improved }}$ and healthy women revealed that some of the IFN signature genes (IFI44, IFI44L, HERC5, CMPK2, SIGLEC1, and MX1) were significantly underexpressed in the pregDAS improved $_{\text {subset compared to }}$ healthy women at T0 $(q<0.05, \mathrm{FC} \geq 2)$. However, by T3, there were no significant differences in expression of these genes between the two groups of women. The IFNinducible genes that were over-expressed at T3 among

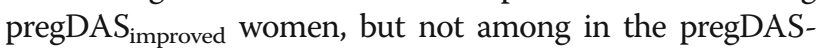
worse women, belong to a common functional network, as shown by known and predicted protein interactions from the STRING database (Fig. 3). Other than RSAD2, none of the genes shown in this network were differentially expressed (T3 vs T0) among healthy women.

\section{Discussion}

There have been no previous reports of pregnancyinduced changes in gene expression in RA or healthy women from pre-pregnancy to the third trimester, other than our own published findings from our pilot RNA-seq dataset [13]. Gene expression changes that accompany improvement or worsening of RA disease activity during pregnancy, relative to a pre-pregnancy baseline, have also not been investigated. Thus, our findings are novel.

In our data, genes showing similar differential expression from $\mathrm{T} 0$ to $\mathrm{T} 3$ in both the pregDAS improved $_{\text {and }}$ pregDAS worse $_{\text {women }}$ were also similarly differentially expressed among healthy women, suggesting that these were normal pregnancy-related changes. The observation that a cluster of type I IFN-inducible genes demonstrated contrasting changes in expression from $\mathrm{T} 0$ to $\mathrm{T} 3$ between the pregDAS $S_{\text {improved }}$ and pregDAS $S_{\text {worse }}$ women was particularly interesting. These genes were over-expressed at T3 among the pregDAS $S_{\text {improved }}$ women, compared to the T0 baseline, even though at T3 five of the eight pregDAS $S_{\mathrm{im}}$ proved women were on corticosteroid therapy, which has been shown to inhibit type I IFN signaling [23, 24]. Over-expression of IFN-inducible genes has also been observed in RNA-seq profiles of neutrophils among patients with juvenile idiopathic arthritis (JIA) who were in drug-induced remission compared to those with active disease [25]. Neutrophils appear to be activated during pregnancy, as reported by us [13] and others [26-28]. However, neutrophil-related genes were over-expressed in both the pregDAS ${ }_{\text {improved }}$ and pregDAS ${ }_{\text {worse }}$ groups; yet, levels of the IFN-inducible genes (except IFI27) decreased from T0 to T3 in the pregDAS ${ }_{\text {worse }}$ subset (not significantly). Thus, over-expression of IFN-inducible genes during pregnancy among pregDAS $S_{\text {improved }}$ women may not be due to 


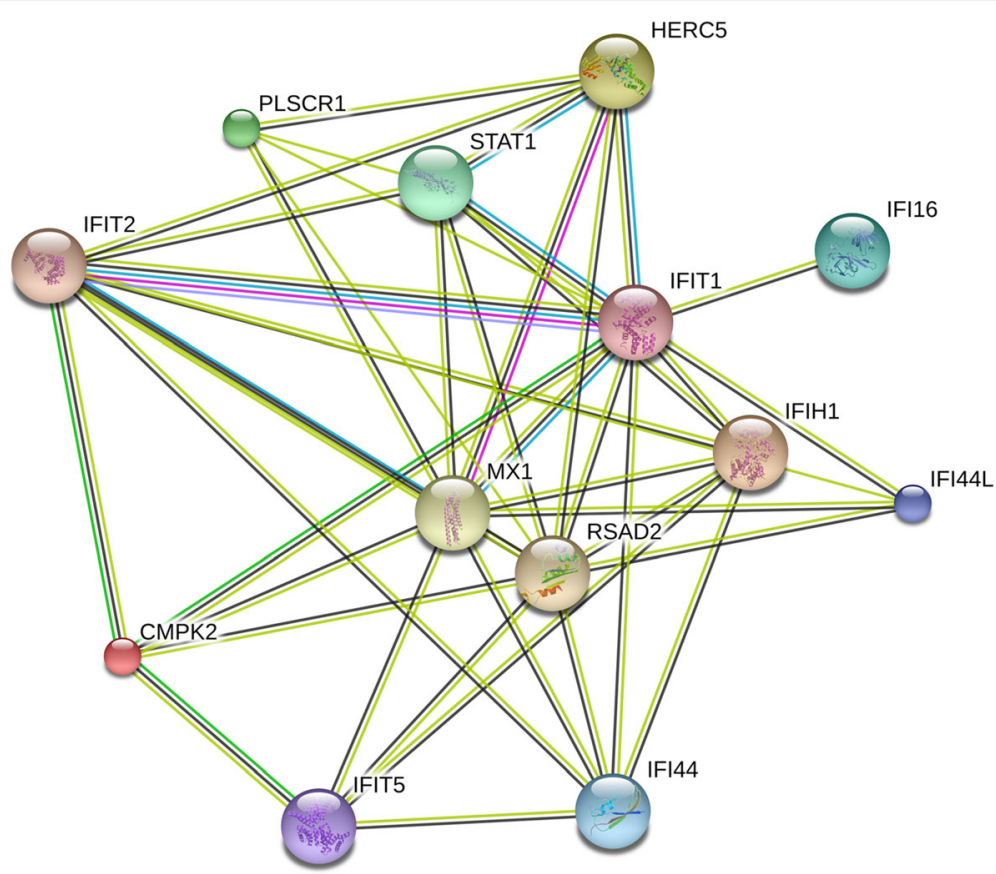

Fig. 3 Network graph showing associations between proteins encoded by genes with contrasting changes in expression from the pre-pregnancy baseline to the third trimester between RA women who improved and those who worsened. The nodes in the network represent proteins encoded by genes. The edges represent known as well as predicted interactions between the proteins, suggesting that they are part of a common functional network. Proteins that were not joined in the network are not shown. Variations at the levels of transcripts or post-translational modification are also not shown. Nodes with unknown 3D structure are shown by smaller size

neutrophil activation. Of interest, the three women who worsened during pregnancy were all on anti-TNF therapy at baseline, and non-responders to anti-TNF therapy have been reported to produce lower levels of IFN $\gamma$ than good responders [29, 30]. Although we might also expect type I IFN genes to be differentially expressed in concert with type I IFN-inducible genes, we found no such evidence in our data.

The significance of the type I IFN signature in the

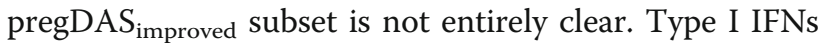
appear to have conflicting roles in autoimmune diseases. Whereas a type I IFN signature in lupus is associated with active disease [31], it correlates with an alleviation of symptoms in multiple sclerosis (MS) [32], and IFN $\beta$ therapy has been used successfully to treat patients with MS [33]. In vitro studies as well as studies of animal models of arthritis suggest that type I IFN most likely has a protective role in RA [32, 34], although translation of the findings from the animal models to treat human RA using IFN $\beta$ therapy has thus far not been successful [34]. The majority of the IFN-inducible genes identified in our data were under-expressed in the pregDAS improved $_{1}$ subset compared to healthy women at T0, which may suggest that expression patterns of these genes in RA could be deregulated as a consequence of the disease. Type I IFNs appear to promote down-regulation of pro- inflammatory cytokines and up-regulation of antiinflammatory cytokines in organ-targeted autoimmune diseases [32, 35]. It is thus possible that the lower levels of IFN-inducible genes in the pregDAS $S_{\text {improved }}$ women before pregnancy may reflect lower systemic IFN $\beta$ levels, which favor a pro-inflammatory state. Furthermore, while expression levels of the IFN-inducible genes did not change significantly from T0 to T3 among healthy women, they changed significantly in the pregDAS $S_{\text {improved }}$ women, and by T3, they were no longer differentially expressed between the two groups (data not shown). These observations support the notion that increased levels of the IFNinducible genes identified in our pilot data may help shift the immunomodulatory balance to an anti-inflammatory state during pregnancy. We speculate that specific levels of these gene products may be crucial to maintaining a healthy pregnancy, explaining why expression levels at T3 among pregDAS $\mathrm{S}_{\text {improved }}$ women became comparable to those of healthy women.

Some of the IFN-inducible genes that were overexpressed at $\mathrm{T} 3$ in our data (IFI44, IFI44L, and SIGLEC1) have been reported to show increased expression in pregnant RA women compared to unrelated non-pregnant RA women [12]. That study found no association between expression levels of these genes and disease activity. However, pre-pregnancy 
data on both disease activity and expression levels were not available for comparison with pregnancy data. Although in the present study we did not test for associations between expression of IFN-inducible genes and disease activity, we cannot exclude the possibility that they may be involved.

The strengths of our study include the availability of paired time-dependent data from the same women at both time points. This allowed global gene expression changes induced by pregnancy to be compared to a prepregnancy baseline, while at the same time controlling for unmeasured confounders. The use of RNA-seq technology to assess gene expression was also an advantage over microarray data. Our study does have some limitations. First, the sample sizes were small, especially for the women who worsened during pregnancy. However, the availability of data from the same women before and during pregnancy, as well as the ethnic homogeneity of the study population, enabled us to overcome some of the limitations of having a small sample size. Nevertheless, observations relating to the pregDAS ${ }_{\text {worse }}$ subset should be interpreted with caution until they can be replicated in a larger sample. Because samples were processed in two batches, we used sample replicates in both batches to assess and mitigate batch effects. We did not examine changes in proportions of different cell types in blood samples across time points, because our goal was to identify overall systemic gene expression changes resulting either from altered expression of specific genes or from differences in cell proportions. Although there is a possibility that anti-TNF and/or other medications may have influenced the results, the lack of variation in medication use within each subset of women with RA precluded us from determining if this was the case. Nevertheless, in the pregDAS $S_{\text {improved }}$ group, exclusion of the woman on anti-TNF therapy did not significantly change the results, suggesting that the findings were not influenced by anti-TNF. We did not adjust for dosage and/or specific medications.

\section{Conclusions}

The results from our pilot study suggest that genes demonstrating significant changes in expression in the pregDAS $_{\text {improved }}$ women, but not the pregDAS ${ }_{\text {worse }}$ women, during pregnancy - that is, those showing similar patterns of expression as in healthy pregnancy or those among the IFN signature - could be involved in the natural amelioration of RA. These findings warrant further investigations into expression of these genes in RA pregnancy, but are nevertheless preliminary, and should be interpreted with caution until replicated in a larger sample.

\section{Additional files}

\begin{abstract}
Additional file 1: Table S1. Within-group differential expression results (T3 vs T0) for the 161 genes that showed significant differential expression $(q<0.05, F C \geq 2)$ among the pregDAS improved women. Fold changes (FCs) in expression, $p$ values (unadjusted), and $q$ values are shown for each of the three groups of women (i.e., pregDAS improved, pregDAS worse, and healthy women). Given the small sample sizes, we recommend that the $q$ values be interpreted with caution, especially among the three pregDAS worse women. (PDF $153 \mathrm{~kb}$ )

Additional file 2: Figure S1. Numbers of genes differentially expressed $(q<0.05, F C \geq 2)$ in the three groups of women. (Note: These numbers are provided only to give context, given the small sample sizes). (PDF $5 \mathrm{~kb}$ )
\end{abstract}

\begin{abstract}
Abbreviations
CRP: C-reactive protein; DAS28-CRP4: Disease Activity Score based on 28 joint counts and 4 variables, including C-reactive protein ; FC: Fold-change; GH: Patient global health; GLM: Generalized linear model; GO: Gene Ontology; IFN: Interferon; JIA: Juvenile idiopathic arthritis; MS: Multiple sclerosis; pregDAS improved: Women with rheumatoid arthritis whose disease activity improved during pregnancy; pregDAS worse: Women with rheumatoid arthritis whose disease activity worsened during pregnancy; RA: Rheumatoid arthritis; RNA-seq: RNA sequencing; rRNA: Ribosomal RNA; SJC28: Swollen joint count based on 28 joints; T0: Pre-pregnancy; T3: Third trimester; TJC28: Tender joint count based on 28 joints; TMM: Trimmed Mean of M values; TNF: Tumor necrosis factor
\end{abstract}

\section{Acknowledgements}

We are immensely grateful to the study subjects for their participation in the study. We thank Mr. Kurt Stig Jensen at the Juliane Marie Center for his support. The Rheumatology departments at the following hospitals in Denmark facilitated collection of data and samples:

Rigshospitalet (Glostrup), Odense Universitetshospital, Kong Christian X's Gigthospital (Gråsten), Aarhus University Hospital NBG, and

Regionshospitalet Viborg. We thank all members of our project team for making this work possible: Anne-Grethe Rasmussen, Charlotte Schön Frengler, Dorte Heide, Randi Petersen, Tove Thorup Rasmussen, Lone Thomasen, Britta Hvidberg Nielsen, Teresa Rozenfeldt, Kirsten Junker, Lis Kastberg Schubert, Lis Lund, Jette Barlach, Helle Bendtsen, Helle Andersen, and Marjo Westerdahl for their contribution to data and sample collection; and Rikke Godtkjær Andersen, Mie Rams Rasmussen, Pia Pedersen, Stine Birkelund, Louise Mielke, and Andreas Smed for management of data and samples. We also greatly appreciate the valuable assistance provided by Majbritt Norman Nielsen and DANBIO personnel.

\section{Funding}

This work was supported in part by funds from the National Institute of Arthritis, Musculoskeletal and Skin Diseases (NIAMS), USA (grant R21 AR057931); Gigtforeningen, Denmark (grant R87-A1477-B512); and the Juliane Marie

Center, Rigshospitalet (Denmark). These funders did not have any role in conducting this study or in the interpretation and reporting of results.

\section{Availability of data and materials}

The data are governed by Danish privacy laws. The authors are legally forbidden from publicly sharing data under the terms of their agreement with the Danish Data Protection Agency. Data are available upon request from the corresponding author, after approval is granted by the Danish Data Protection Agency.

\section{Authors' contributions}

DEG analyzed the data, interpreted the results, and contributed to manuscript writing. MKS was responsible for acquisition of the data. LP and EP contributed to the analysis and interpretation of the data. JLN, HK, and JO contributed to the conception and design of the study. HK was also responsible for data acquisition. $\mathrm{MLH}, \mathrm{VZ}$, and $\mathrm{BO}$ contributed to the data acquisition. DJ was involved in the conception and design of the experiments, in the analysis and interpretation of the data, and in writing the manuscript. All authors contributed to critically revising the manuscript for important intellectual content. All authors read and approved the final 
manuscript. HK passed away before the submission of the final version of the manuscript; DJ accepts responsibility for the integrity and validity of the data collected and analyzed.

\section{Competing interests}

The authors declare that they have no competing interests.

\section{Consent for publication}

Not applicable.

\section{Ethics approval and consent to participate}

This study was approved by the ethics committee for Region Hovedstaden (Denmark), the Danish Data Protection Agency, and the Children's Hospital Oakland Research Institute Institutional Review Board. All subjects provided written informed consent prior to enrollment.

\section{Publisher's Note}

Springer Nature remains neutral with regard to jurisdictional claims in published maps and institutional affiliations.

\section{Author details}

'UCSF Benioff Children's Hospital Oakland, Children's Hospital Oakland Research Institute, 5700 Martin Luther King Jr. Way, Oakland, CA, USA. ${ }^{2}$ University of California, Berkeley, Berkeley, CA, USA. ${ }^{3}$ Juliane Marie Center, Copenhagen University Hospital, Rigshospitalet, Copenhagen, Denmark. ${ }^{4}$ California Institute of Technology, Pasadena, CA, USA. ${ }^{5}$ Fred Hutchinson Cancer Research Center, Seattle, WA, USA. 'University of Washington, Seattle, WA, USA. ${ }^{7}$ University of California, Los Angeles, Los Angeles, CA, USA. ${ }^{8}$ Aarhus University, Aarhus, Denmark. ${ }^{9}$ DANBIO Registry and Copenhagen Centre for Arthritis Research, Centre for Rheumatology and Spine Diseases (VRR), Rigshospitalet, Glostrup, Denmark. ${ }^{10}$ Faculty of Health and Medical Sciences, University of Copenhagen, Copenhagen, Denmark. ${ }^{11}$ University of California, San Francisco, San Francisco, CA, USA.

\section{Received: 16 January 2017 Accepted: 2 May 2017}

\section{Published online: 25 May 2017}

\section{References}

1. Cross M, Smith E, Hoy D, Carmona L, Wolfe F, Vos T, et al. The global burden of rheumatoid arthritis: estimates from the global burden of disease 2010 study. Ann Rheum Dis. 2014;73(7):1316-22.

2. Hench PS. The ameliorating effect of pregnancy on chronic atrophic (infectious rheumatoid) arthritis, fibrositis, and intermittent hydrarthrosis. Mayo Clin Proc. 1938;13:161-7.

3. Nelson $\mathrm{J}$, Ostensen M. Pregnancy and rheumatoid arthritis. Rheum Dis Clin North Am. 1997;23(1):195-212.

4. de Man YA, Dolhain RJ, van de Geijn FE, Willemsen SP, Hazes JM. Disease activity of rheumatoid arthritis during pregnancy: results from a nationwide prospective study. Arthritis Rheum. 2008:59(9):1241-8.

5. Barrett JH, Brennan P, Fiddler M, Silman AJ. Does rheumatoid arthritis remit during pregnancy and relapse postpartum? Results from a nationwide study in the United Kingdom performed prospectively from late pregnancy. Arthritis Rheum. 1999;42(6):1219-27.

6. Nelson JL, Hughes KA, Smith AG, Nisperos BB, Branchaud AM, Hansen JA Maternal-fetal disparity in HLA class II alloantigens and the pregnancy-induced amelioration of rheumatoid arthritis. N Engl J Med. 1993;329(7):466-71.

7. Yan Z, Lambert NC, Ostensen M, Adams KM, Guthrie KA, Nelson JL. Prospective study of fetal DNA in serum and disease activity during pregnancy in women with inflammatory arthritis. Arthritis Rheum. 2006; 54(7):2069-73.

8. Adams Waldorf KM, Nelson JL. Autoimmune disease during pregnancy and the microchimerism legacy of pregnancy. Immunol Invest. 2008;37(5):631-44

9. Haupl T, Ostensen M, Grutzkau A, Burmester GR, Villiger PM. Interaction between rheumatoid arthritis and pregnancy: correlation of molecular data with clinical disease activity measures. Rheumatology (Oxford). 2008;47 Suppl 3:iii19-22.

10. Haupl T, Ostensen M, Grutzkau A, Radbruch A, Burmester GR, Villiger PM. Reactivation of rheumatoid arthritis after pregnancy: increased phagocyte and recurring lymphocyte gene activity. Arthritis Rheum. 2008;58(10):2981-92
11. Weix J, Forger F, Haupl T, Surbek D, Ostensen M, Villiger PM. Influence of pregnancy on the adipocytokine and peroxisome proliferator-activated receptor pathways in peripheral blood mononuclear cells from healthy donors and rheumatoid arthritis patients. Arthritis Rheum. 2012;64(7):2095-103.

12. Weix J, Haupl T, Raio L, Villiger PM, Forger F. The physiologic increase in expression of some type I IFN-inducible genes during pregnancy is not associated with improved disease activity in pregnant patients with rheumatoid arthritis. Transl Res. 2013;161(6):505-12.

13. Mittal A, Pachter L, Nelson JL, Kjaergaard H, Smed MK, Gildengorin VL, et al. Pregnancy-induced changes in systemic gene expression among healthy women and women with rheumatoid arthritis. PLoS One. 2015;10(12):e0145204.

14. Arnett FC, Edworthy SM, Bloch DA, McShane DJ, Fries JF, Cooper NS, et al. The American Rheumatism Association 1987 revised criteria for the classification of rheumatoid arthritis. Arthritis Rheum. 1988;31(3):315-24.

15. Home of the Disease activity score and DAS28. http://www.das-score.nl/.

16. van Gestel AM, Anderson JJ, van Riel PL, Boers M, Haagsma CJ, Rich B, et al. $A C R$ and EULAR improvement criteria have comparable validity in rheumatoid arthritis trials. J Rheumatol. 1999;26(3):705-11.

17. Bray NL, Pimentel $H$, Melsted P, Pachter L. Near-optimal probabilistic RNAseq quantification. Nat Biotechnol. 2016;34(5):525-7.

18. Robinson MD, McCarthy DJ, Smyth GK. edgeR: a Bioconductor package for differential expression analysis of digital gene expression data. Bioinformatics. 2010;26(1):139-40.

19. Robinson MD, Oshlack A. A scaling normalization method for differential expression analysis of RNA-seq data. Genome Biol. 2010;11(3):R25.

20. Zhang B, Kirov S, Snoddy J. WebGestalt: an integrated system for exploring gene sets in various biological contexts. Nucleic Acids Res. 2005;33 Suppl 2:W741-8.

21. von Mering C, Jensen $\sqcup$, Snel B, Hooper SD, Krupp M, Foglierini M, et al. STRING: known and predicted protein-protein associations, integrated and transferred across organisms. Nucleic Acids Res. 2005;33(Database issue):D433-7.

22. Jensen $\amalg$, Kuhn M, Stark M, Chaffron S, Creevey C, Muller J, et al. STRING 8 - a global view on proteins and their functional interactions in 630 organisms. Nucleic Acids Res. 2009;37(Database issue):D412-6.

23. Flammer JR, Dobrovolna J, Kennedy MA, Chinenov Y, Glass CK, Ivashkiv LB, et al. The type I interferon signaling pathway is a target for glucocorticoid inhibition. Mol Cell Biol. 2010;30(19):4564-74.

24. de Jong TD, Vosslamber S, Blits M, Wolbink G, Nurmohamed MT, van der Laken CJ, et al. Effect of prednisone on type I interferon signature in rheumatoid arthritis: consequences for response prediction to rituximab. Arthritis Res Ther. 2015;17:78.

25. Jiang $K$, Sun $X$, Chen $Y$, Shen $Y$, Jarvis JN. RNA sequencing from human neutrophils reveals distinct transcriptional differences associated with chronic inflammatory states. BMC Med Genomics. 2015;8:55.

26. Sacks GP, Studena K, Sargent K, Redman CW. Normal pregnancy and preeclampsia both produce inflammatory changes in peripheral blood leukocytes akin to those of sepsis. Am J Obstet Gynecol. 1998;179(1):80-6.

27. Wang Y, Gu Y, Philibert L, Lucas MJ. Neutrophil activation induced by placental factors in normal and pre-eclamptic pregnancies in vitro. Placenta. 2001;22(6):560-5.

28. Luppi P, Haluszczak C, Trucco M, Deloia JA. Normal pregnancy is associated with peripheral leukocyte activation. Am J Reprod Immunol. 2002;47(2):72-81.

29. Cacciapaglia F, Buzzulini F, Arcarese L, Ferraro E, Afeltra A. The use of an interferon-gamma release assay as a biomarker of response to anti-TNFalpha treatment. Drug Dev Res. 2014;75 Suppl 1:S50-3.

30. Wright HL, Thomas HB, Moots RJ, Edwards SW. Interferon gene expression signature in rheumatoid arthritis neutrophils correlates with a good response to TNFi therapy. Rheumatology (Oxford). 2015;54(1):188-93.

31. Bennett L, Palucka AK, Arce E, Cantrell V, Borvak J, Banchereau J, et al. Interferon and granulopoiesis signatures in systemic lupus erythematosus blood. J Exp Med. 2003;197(6):711-23.

32. Kalliolias GD, Ivashkiv LB. Overview of the biology of type I interferons. Arthritis Res Ther. 2010;12 Suppl 1:S1.

33. Buttmann M, Rieckmann P. Interferon-beta1b in multiple sclerosis. Expert Rev Neurother. 2007;7(3):227-39.

34. Aalbers CJ, Bevaart L, Loiler S, de Cortie K, Wright JF, Mingozzi F, et al. Preclinical potency and biodistribution studies of an AAV5 vector expressing human interferon- $\beta$ (ART-102) for local treatment of patients with rheumatoid arthritis. PLoS One. 2015;10(6):e0130612.

35. Crow MK. Type I interferon in organ-targeted autoimmune and inflammatory diseases. Arthritis Res Ther. 2010;12 Suppl 1:S5. 